\title{
Type I interferon signaling is required for the APOBEC3/Rfv3-dependent neutralizing antibody response but not innate retrovirus restriction
}

\author{
Bradley S. Barrett ${ }^{1}$, Michael S. Harper ${ }^{1,2}$, Sean T. Jones ${ }^{1,2}$, Kejun Guo ${ }^{1}$, Karl J. Heilman ${ }^{1}$, Ross M. Kedl ${ }^{2}$, \\ Kim J. Hasenkrug ${ }^{3}$ and Mario L. Santiago $1,2,4^{*}$ (i)
}

\begin{abstract}
Background: $A P O B E C 3 / R f v 3$ restricts acute Friend retrovirus (FV) infection and promotes virus-specific neutralizing antibody (NAb) responses. Classical Rfv3 studies utilized FV stocks containing lactate-dehydrogenase elevating virus (LDV), a potent type I interferon inducer. Previously, we showed that APOBEC3 is required for the anti-FV activity of exogenous IFN-alpha treatment. Thus, type I interferon receptor (IFNAR) signaling may be required for the APOBEC3/ Rfv3 response.

Results: To test if the APOBEC3/Rfv3 response is dependent on type I IFN signaling, we infected IFNAR knockout versus IFNAR/APOBEC3 double-knockout mice with FV/LDV or LDV-free FV, and evaluated acute FV infection and subsequent NAb titers. We show that LDV co-infection and type I IFN signaling are not required for innate APOBEC3mediated restriction. By contrast, removal of LDV and/or type I IFN signaling abrogated the APOBEC3-dependent NAb response.

Conclusions: $\mathrm{APOBEC} 3$ can restrict retroviruses in a type I IFN-independent manner in vivo. By contrast, the ability of APOBEC3 to promote NAb responses is type I IFN-dependent. These findings reveal novel insights on the interplay between type I IFNs and APOBEC3 in vivo that may have implications for augmenting antiretroviral NAb responses.
\end{abstract}

Keywords: IFNAR, Friend retrovirus, Neutralizing antibody, Deaminase, LDV

\section{Background}

Innate immune mechanisms provide a means for the host to control pathogens before more slowly developing adaptive immune responses come into play. Innate immunity was linked to the production of type I interferons (IFN), which orchestrate an antiviral state through the expression of hundreds of interferon-stimulated genes (ISGs) [1]. Some ISGs encoded proteins known as 'restriction factors', which directly inhibit invading pathogens. These restriction factors include the seven

\footnotetext{
*Correspondence: mario.santiago@ucdenver.edu

${ }^{4}$ Division of Infectious Diseases, University of Colorado Denver, Mail Stop

B-168, 12700 E 19th Avenue, Aurora, CO 80045, USA

Full list of author information is available at the end of the article
}

human APOBEC3 enzymes (hA3A to hA3H): deoxycytidine deaminases that counteract a broad range of retroviruses including HIV-1 [2, 3], and exhibit a wide range of sensitivities to type I IFN induction [4-7]. In the absence of the HIV-encoded antagonist Vif, APOBEC3 gets incorporated into budding HIV-1 particles, inhibiting replication in the next target cell either by physically impeding reverse transcription or hypermutating singlestranded reverse transcripts [8]. However, the majority of APOBEC3 studies involved the ectopic expression of APOBEC3 in cell lines, which may not be physiologically relevant. In particular, transfecting APOBEC3 expression constructs into cells would bypass potential upstream regulatory pathways such as type I IFN signaling that 
may be required for APOBEC3 to restrict retroviral replication in vivo.

In contrast to humans, mice encode only one APOBEC3 gene, mA3. Thus, mA3 knockout (KO) mice provided the field with a powerful means to understand the in vivo impact of APOBEC3 on retroviral pathogenesis and immunity [9-12]. Our group utilized the murine Friend retrovirus (FV) infection model, which was key to the identification of host genes that control retrovirus infection, including the first retrovirus restriction factor, Fv1 $[13,14]$. FV is a complex of a replication-competent Friend murine leukemia virus (F-MuLV) and a replication-defective spleen-focus forming virus (SFFV) that causes splenomegaly and erythroleukemia [15]. We previously showed that C57BL/6 (B6) wild-type (WT) and mA3 KO mice had similar levels of plasma viral load at 7 days post-infection (dpi), but the infectivity of the virions was significantly higher in mA3 KO mice [10, 16-18]. Importantly, administration of recombinant IFN $\alpha$ inhibited FV replication in WT mice, but exogenously administered IFN $\alpha$ had no antiviral effect in $\mathrm{mA} 3 \mathrm{KO}$ mice [17]. These findings indicated that $\mathrm{mA} 3$ acts downstream of exogenous IFN $\alpha$ to inhibit FV replication. However, it remains unclear if $\mathrm{mA} 3$ can inhibit retroviral infection in vivo in the absence of endogenous type I IFN signaling.

The impact of $\mathrm{mA} 3$ extends beyond retrovirus restriction. Our group and others reported that $\mathrm{mA} 3$ encoded $\mathrm{Rfv} 3$, a classical resistance gene in $\mathrm{B} 6$ mice that promoted recovery from $\mathrm{FV}$ viremia by stimulating a stronger $\mathrm{NAb}$ response $[10,11,19,20]$. These early studies on Rfv3 were conducted in $\mathrm{F}_{1}$ hybrid mice harboring the $F v 2$ susceptibility allele from BALB or A.BY strains, which promotes higher FV replication by driving erythroblast proliferation [21-23]. Nevertheless, the impact of mA3/ $\mathrm{Rfv} 3$ on NAb responses was also observed in pure B6 mice. Compared to B6 WT mice, B6 mA3 KO mice had significantly lower FV-specific NAb responses by 28 days post-infection (dpi) [10]. The underlying mechanism was multifaceted. Compared to $\mathrm{mA} 3 \mathrm{KO}$ mice, WT mice exhibited: (1) enhanced germinal center (GC) responses due to noninfectious virion particle release [16]; (2) augmented $\mathrm{GC}$ responses due to contraction of the marginal zone B cell compartment [24]; (3) higher levels of antiviral IgG2b and IgG2c antibodies [25]; and (4) enhanced somatic hypermutation of virus-specific IgG antibodies [26]. Antibody neutralization was dependent on Fcy receptors, as removal of the common $\gamma$ chain $(\mathrm{FcR} \gamma)$ and particularly Fc $\gamma$ RIV, which bind to IgG2b and IgG2c antibodies, rendered $28 \mathrm{dpi}$ antisera incapable of neutralizing $\mathrm{FV}$ in vivo [25]. By contrast, removal of complement C3 had no effect on the in vivo neutralization capacity of 28 dpi antisera from mA3-sufficient mice [25].
Interestingly, Rfv3 was initially discovered using FV stocks that contained lactate-dehydrogenase elevating virus (LDV), an endemic RNA virus in wild mouse populations and component of the murine 'virome' [27, 28]. Since Rfv3 was discovered using FV/LDV stocks, our studies on the role of $\mathrm{mA} 3$ in NAb responses utilized FV/ LDV $[10,16,20,26]$. LDV has potent immunostimulatory properties; thus, data obtained using FV/LDV may not necessarily be reproduced using 'LDV-free' FV. LDV can suppress $\mathrm{T}$ and $\mathrm{B}$ cell responses in FV infection [29-31], and can induce high levels of type I IFNs through Tolllike receptor 7 (TLR7) sensing [32]. LDV-free FV infection of B6 mice resulted in very low or undetectable IFNa expression compared to FV/LDV co-infection [33]. Notably, type I IFNs can also augment and shape humoral immune responses in vivo [34-37]. In certain contexts, LDV may also enhance antibody responses [38, 39]. Thus, we hypothesized that type I IFN signaling might be required for the $\mathrm{mA3} / \mathrm{Rfv} 3$-dependent NAb response during FV/LDV infection.

In order to investigate the impact of type I IFN signaling in $\mathrm{mA} 3$ restriction and $\mathrm{NAb}$ responses, we prepared mice doubly-deficient in $\mathrm{mA} 3$ and the type I IFN receptor (IFNAR). IFNAR is a heterodimer consisting of IFNAR-1 and IFNAR-2 subunits that together form a binding site for the antiviral cytokines IFN $\beta$ and IFN $\alpha$ subtypes [40]. IFNAR KO mice lacked the IFNAR-1 receptor chain and were unresponsive to JAK/STAT signaling cascades triggered by type I IFNs [41]. Many viral infections, including FV, replicated to significantly higher levels in IFNAR KO compared to WT mice $[41,42]$. However, the downstream effector mechanisms remain under intense investigation. In this report, we tested whether $\mathrm{mA} 3$ can inhibit FV and FV/LDV infection and promote $\mathrm{NAb}$ responses in the absence of IFNAR signaling.

\section{Results}

Murine APOBEC3 inhibited infectious virus release in the absence of type I IFN signaling

We previously demonstrated that $\mathrm{B} 6 \mathrm{~mA} 3 \mathrm{KO}$ mice had higher infectious viremia compared to wild-type (WT) mice in experimental infections using LDV-containing FV stocks (FV/LDV) [10, 16] or LDV-free FV stocks (FV) $[18,43]$. These data suggested that the induction of type I IFNs by LDV might not be required for $\mathrm{mA3}$ to inhibit FV infection in vivo. To test this hypothesis directly, IFNAR KO and IFNAR/mA3 double KO (dKO) mice $\left(<1\right.$ year old) were infected i.v. with $10^{4}$ spleen focus forming units (SFFU) of FV/LDV or FV (Fig. 1a). At 7 days post-infection (dpi), infectious viremia in the plasma was evaluated. Plasma infectious viremia was 5 to eightfold higher in IFNAR/mA3 dKO mice compared to IFNAR KO mice in both FV/LDV (Fig. 1b, left panel) 


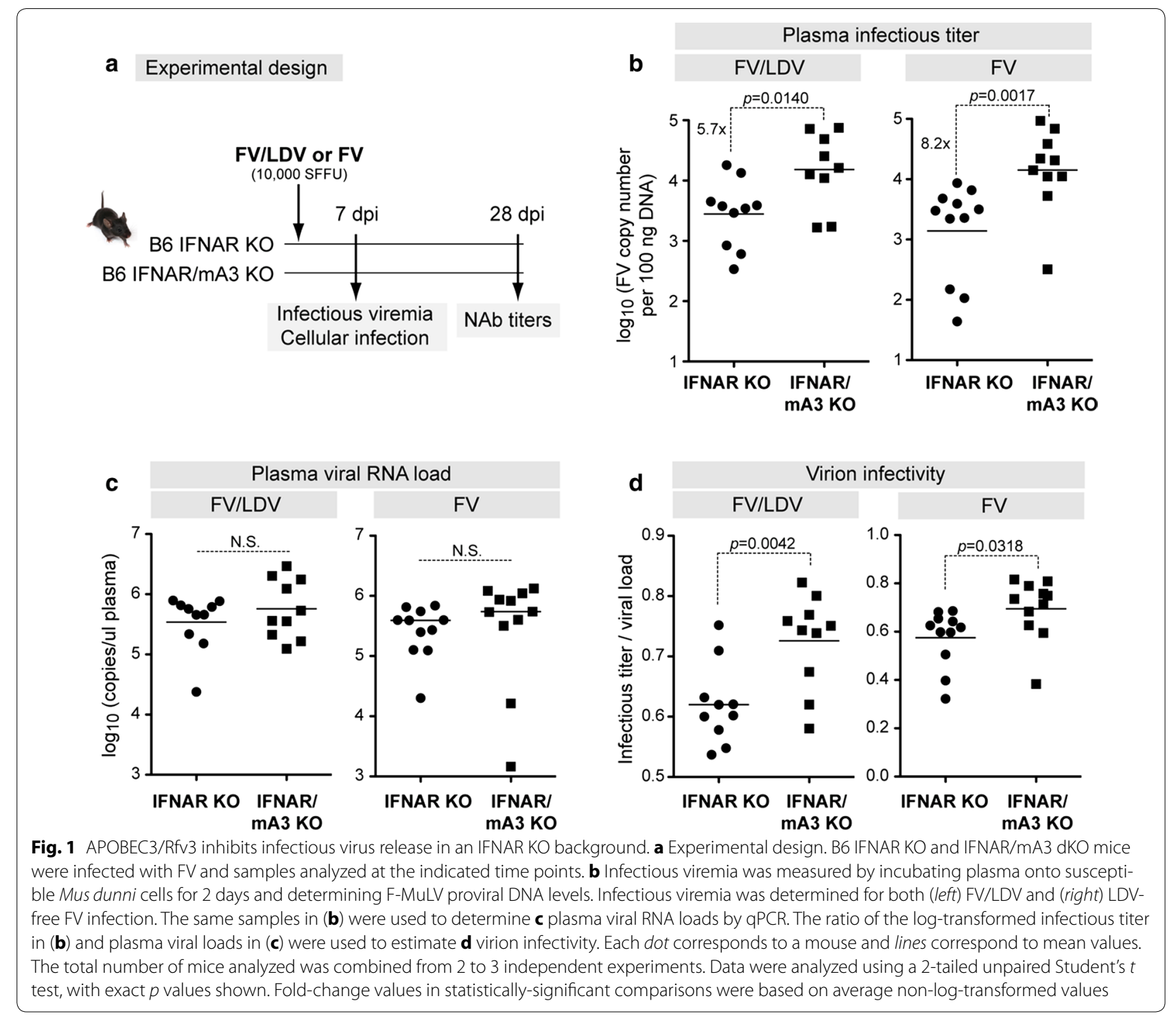

and FV (Fig. 1b, right panel) infections. We next quantified the levels of viral RNA in the plasma (Fig. 1c), an indirect measure of the total number of particles released. Consistent with our previous findings on WT versus $\mathrm{mA} 3 \mathrm{KO}$ mice [17], we found no significant difference in plasma viral RNA load between IFNAR and IFNAR/mA3 dKO mice at $7 \mathrm{dpi}$ in both FV/LDV and FV infections (Fig. 1c). The ratio of infectious titers (Fig. 1b) and plasma viral RNA load (Fig. 1c) provides a measure of virion infectivity $[16,17]$. As shown in Fig. 1d, virions in the $7 \mathrm{dpi}$ plasma of IFNAR/mA3 dKO mice had significantly higher infectivity than those from IFNAR KO mice. Our findings reveal that type I IFN signaling and LDV co-infection are not required for mA3-mediated inhibition of virion infectivity during acute infection.
IFNAR signaling is not required for $m A 3$ restriction of acute FV/LDV or FV infection

To complement the plasma viremia results, we evaluated cellular F-MuLV infection levels in splenocytes. To detect F-MuLV infected cells, we utilized a previously described flow cytometry method using MAb 34, which is specific for the F-MuLV glyco-gag protein [16, 44] (Fig. 2a). Flow cytometry-based methods to detect virus infection generally have lower dynamic range than quantitative PCRbased methods. Nevertheless, consistent with the plasma infectious viremia data, we observed that in both FV/ LDV (Fig. 2b) and FV (Fig. 2c) infections, mA3 deficiency in IFNAR KO mice resulted in significantly higher splenocyte infection levels (1.6-fold) compared to IFNAR KO mice. Altogether, the data in Figs. 1 and 2 indicate that 


\section{a Quantifying FV infected cells by flow cytometry}

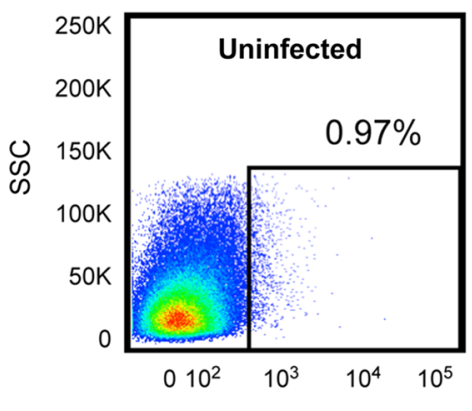

Glyco-Gag+

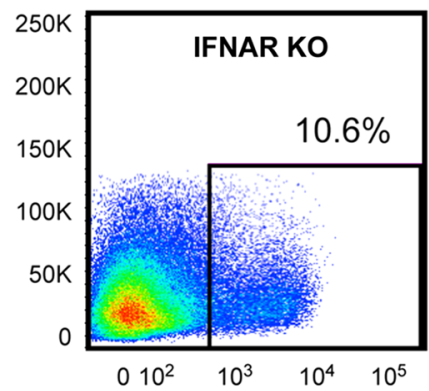

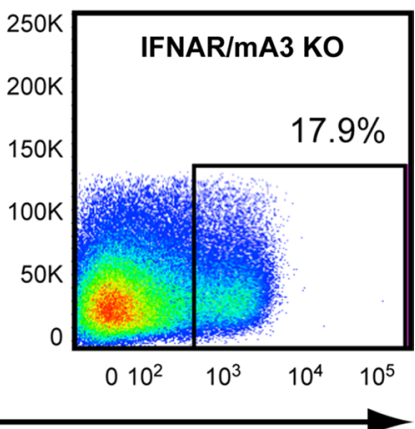

FV b

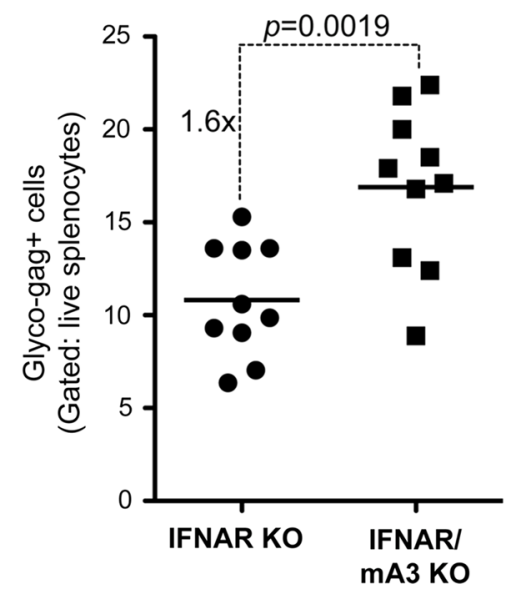

C

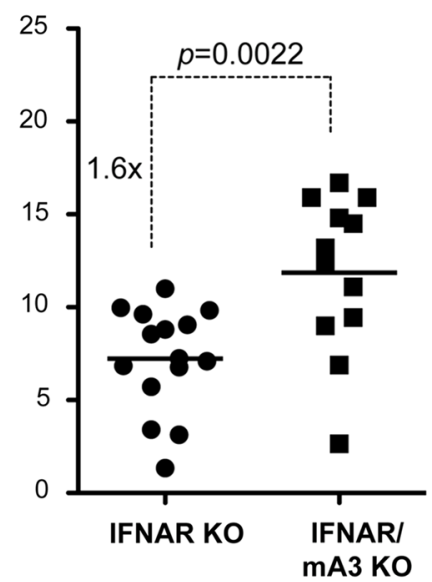

Fig. 2 APOBEC3/RfV3 inhibits acute FV infection of splenocytes independent of type I IFN signaling. Splenocyte FV infection levels were measured by flow cytometry using a glyco-gag specific monoclonal antibody. a Representative flow plots showing glyco-gag+ splenocytes from FV/LDV infected mice at $7 \mathrm{dpi}$. The percentage of live splenocytes that expressed glyco-gag was evaluated in $\mathbf{b}$ FV/LDV and $\mathbf{c} L D V$-free FV infections. Each dot corresponds to a mouse and lines correspond to mean values. The total number of mice analyzed was combined from 2 to 3 independent experiments. Fold-change of mean values per cohort are shown. Data were analyzed using a 2-tailed unpaired Student's $t$ test, with exact $p$ values shown

$\mathrm{mA} 3$ restricted acute FV replication independently of type I IFN signaling and LDV co-infection.

\section{LDV co-infection is required for the $\mathrm{mA} 3 / \mathrm{Rfv} 3$ neutralizing antibody phenotype}

The Rfv3 gene was identified using FV/LDV stocks [21]. LDV is a potent stimulator of type I IFN responses [32], and type I IFNs can stimulate B cell responses [34-37, 45]. To test if mA3/Rfv3-mediated enhancement of $\mathrm{NAb}$ responses occurs in LDV-free FV infection, WT and $\mathrm{mA} 3 \mathrm{KO}$ mice were infected with $10^{4} \mathrm{SFFU}$ of FV/ LDV or FV and plasma NAb levels were evaluated at 28 dpi. Consistent with our previously published data [10, 25], mA3 $\mathrm{KO}$ mice had significantly lower NAb titers (3.4-fold) compared to WT mice during FV/LDV coinfection (Fig. 3a). By contrast, the difference in NAb responses between WT and $\mathrm{mA} 3 \mathrm{KO}$ mice infected with LDV-free FV was not statistically significant (Fig. 3b). Thus, LDV co-infection is required for $\mathrm{mA} 3$ to promote the FV-specific NAb response.

\section{IFNAR signaling is required for the $\mathrm{mA3} / \mathrm{Rfv} 3$-dependent neutralizing antibody response}

Since type I IFNs can influence antibody responses and LDV is a potent inducer of type I IFNs, we next investigated if type I IFN signaling is required for the $\mathrm{mA} 3$ / Rfv3-dependent NAb response. IFNAR KO and IFNAR/ mA3 KO mice were infected with $10^{4} \mathrm{SFFU}$ of FV/LDV 

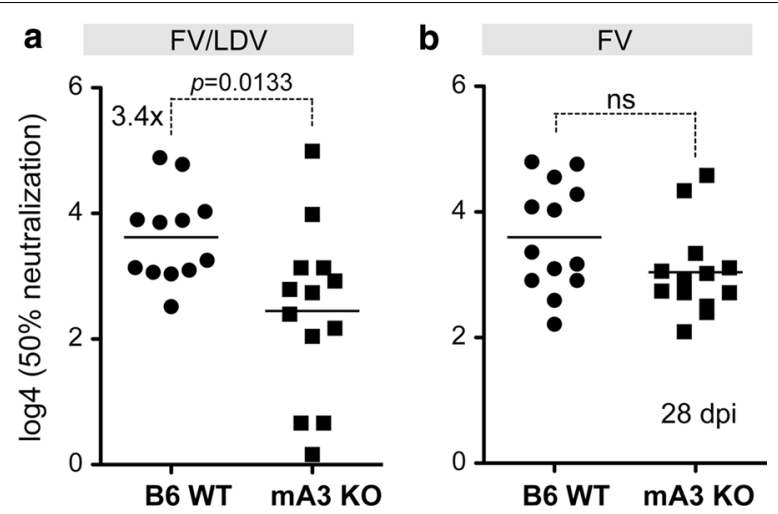

Fig. 3 LDV co-infection is critical for the APOBEC3/RfV3-dependent NAb response. B6 WT and $\mathrm{mA} 3 \mathrm{KO}$ mice were infected with $10^{4} \mathrm{SFFU}$ of $\mathbf{a}$ FV/LDV or $\mathbf{b}$ LDV-free FV. Plasma samples at $28 \mathrm{dpi}$ were heatinactivated and the reciprocal plasma dilution that conferred 50\% neutralization was computed. $\log _{4}$-transformed data are shown and used for statistical analyses. Each dot corresponds to a mouse and lines correspond to mean values. The total number of mice analyzed was combined from 2 independent experiments. Fold-change values in statistically-significant comparisons were based on median nonlog-transformed values. Data were analyzed using 2-tailed unpaired Student's $t$ test, with $p$ values indicated; ns, not significant $(p>0.05)$

and NAb titers evaluated from 28 dpi plasma. In contrast to FV/LDV infections showing that $\mathrm{mA} 3$ influences $\mathrm{NAb}$ responses in the B6 background (Fig. 3a) [10, 25], the NAb titers between IFNAR $\mathrm{KO}$ and IFNAR/mA3 $\mathrm{KO}$ mice were not significantly different from each other (Fig. 4a).

We previously showed that the mA3-dependent NAb response correlated with higher titers of FV-specific IgG2b and IgG2c, both of which signal through FcyRIV $[25,46]$. We therefore quantified FV-specific endpoint IgG2 titers at 28 dpi plasma of FV/LDV-infected IFNAR $\mathrm{KO}$ versus IFNAR/mA3 $\mathrm{KO}$ mice. In contrast to $\mathrm{mA} 3$ influencing antiretroviral IgG2b and IgG2c titers, mA3 deficiency had no impact on the FV-specific IgG2 response in an IFNAR KO background (Fig. 4b). These data suggest that type I IFN signaling modulates the APOBEC3/Rfv3-dependent, FV-specific IgG2b/c response.

IFNAR KO mice are more susceptible to FV infection compared to normal B6 mice [42]. IFNAR KO mice infected with $10^{4}$ SFFU of FV had viral loads that were 250-fold higher than B6 WT mice infected with the same inoculum dose. Thus, the observation that mA3 can influence NAb responses in the B6 background (Fig. 3a) but not in an IFNAR KO background (Fig. 4a) may be due to a difference in infection levels and/or antigen load. Specifically, higher FV titers may result in greater immunosuppression in the IFNAR KO background. To address this caveat, we infected IFNAR KO mice with fivefold lower FV inoculum dose (2000 SFFU) and evaluated NAb titers at $28 \mathrm{dpi}$. A 2000 SFFU inoculum resulted in FV viral loads in IFNAR KO mice that were comparable to that of B6 WT mice infected with $10^{4}$ SFFU at $7 \mathrm{dpi}$ $\left(\leq 10^{3}\right.$ copies/ml of F-MuLV). At this lower inoculum dose, we did not observe a significant difference in NAb titers (Fig. 4c) and FV-specific IgG2b/c titers (Fig. 4d) between IFNAR KO and IFNAR/mA3 double-KO mice. Combining the results from Figs. 3 and $4 \mathrm{c}$ in a four-way comparison, removal of IFNAR signaling in mA3 $\mathrm{KO}$ mice did not lead to a further decrease in NAb responses (Additional file 1: Fig. S1). This data suggested that IFNAR and mA3 function in a linked pathway controlling $\mathrm{NAb}$ responses. Altogether, the data demonstrates that the mA3-dependent antibody response against FV/LDV infection requires type I IFN signaling in vivo.

\section{Discussion}

Type I IFN signaling is required for controlling many viral infections, but the downstream mechanisms remain less understood. Recently, major efforts have been undertaken to determine which of the hundreds of ISGs act as antiviral effectors in vivo. Several APOBEC3 genes are considered as ISGs. Thus, we hypothesized that APOBEC3 may be a critical effector of endogenous type I IFN response against FV infection. Surprisingly, we found that mA3 acts as a type I IFN-independent restriction factor that limits acute FV infection. A likely explanation is that B6 mice already express high baseline expression levels of mA3 [47-49], potentially mitigating the need for type I IFN-mediated induction of mA3 to achieve restriction. However, the result is intriguing given that $\mathrm{mA} 3$ is critical for the inhibitory activity of recombinant IFN $\alpha$ treatment against FV infection in vivo [17]. Collectively, our findings suggest that the effector mechanisms mobilized by exogenous IFN $\alpha$ administration (primarily APOBEC3) might be different from that of an endogenous type I IFN response (APOBEC3 + other ISGs) during retrovirus infection. We speculate that the difference in ISG effector(s) mobilized during exogenous IFN $\alpha$ treatment versus the endogenous type I IFN response may be due to the nature of the type I IFNs involved. Our previous study involving exogenous IFN $\alpha$ treatment utilized only one type of IFN $\alpha$ (universal) [17], but an endogenous type I IFN response likely stimulates a combination of multiple IFN $\alpha$ subtypes. The IFN $\alpha$ subtypes demonstrate diverse biological properties, including antiviral activities, both in vitro and in vivo $[50,51]$. To date, it remains unclear if diverse IFN $\alpha$ subtypes may be stimulating distinct antiviral effectors in vivo. The antiviral ISG effectors that act downstream of IFNAR signaling to reduce FV replication in vivo remains to be determined. 


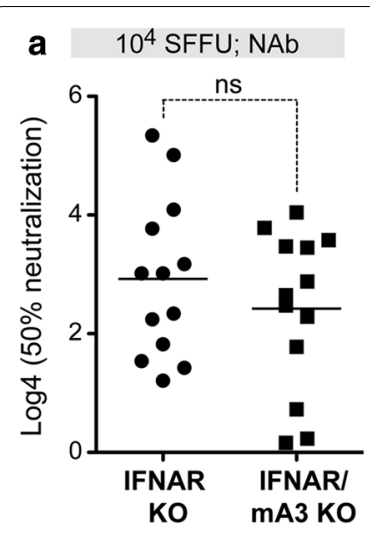

b $\quad 10^{4} \mathrm{SFFU;} \operatorname{IgG} 2 \mathrm{~b} / \mathrm{c}$
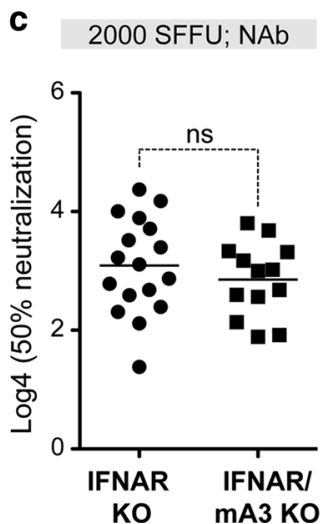

Fig. 4 APOBEC3/Rfv3-dependent NAb response requires type I IFN signaling. Mice were infected with FV/LDV at two different inoculum doses: a, b 10,000 SFFU and c, d 2000 SFFU. a, c Plasma samples at 28 dpi were heat-inactivated and the reciprocal plasma dilution that conferred $50 \%$ neutralization was computed. Log $_{4}$-transformed data are shown and used for statistical analyses. b, d FV-specific lgG2b/c titers were determined by endpoint-titration ELISA for mice infected with $10^{4}$ SFFU of FV/LDV. Native FV virions were coated into ELISA plates and twofold dilutions of plasma were added. IgG2b/c antibodies were detected using a combination of anti-lgG2b and anti-lgG2c conjugates. In all panels, each dot corresponds to a mouse and lines correspond to mean values. The total number of mice analyzed was combined from 2 independent experiments. Data were analyzed using 2-tailed unpaired Student's $t$ test; ns not significant $(p>0.05)$

In contrast to mA3 functioning as a type I IFN-independent restriction factor that limits acute infection, mA3 functioned as a type I IFN-dependent innate resistance factor that promotes virus-specific NAb and IgG2 responses. The reason for why $\mathrm{mA} 3$ requires type I IFN signaling to modulate antibody responses but not retroviral restriction may be due to differences in the complexity of these processes. Retrovirus restriction requires only the APOBEC3 enzyme, whereas orchestrating a $\mathrm{NAb}$ response would require the mobilization of multiple pathways. For example, type I IFNs can modulate the contribution of follicular B cells to the antibody response, resulting in higher antigen-specific IgG2c titers [45].
LDV, a potent type I IFN inducer, was also required to reveal the Rfv3 phenotype. At first glance, this finding appears to be at odds with data showing that LDV can suppress B cell responses during FV infection [31]. FV/ LDV infections exhibited a delay in NAb development compared to LDV-free FV infections in B6 mice [31]. However, this previous study utilized fivefold to tenfold lower levels of viral inoculum compared to our current study. In fact, early studies suggested that under some conditions, LDV may also enhance antibody responses $[38,39]$. Thus, we speculate that at low inoculum doses, LDV may suppress adaptive immune responses. By contrast, at high inoculum doses (such as the one we used in this study), LDV's immunosuppressive effects may have been overcome. Interestingly, FV on its own can suppress $B$ cell responses through PTEN-mediated inhibition of the PI3K pathway [52]. Further studies should help shed insight on how FV and LDV inoculum dose affects the balance between B cell stimulation and suppression in the FV/LDV coinfection model. Overall, the IFNAR-dependence of the mA3/Rfv3 NAb response in the current study suggested that the type I IFN response induced by LDV also activated mA3. This raises the possibility that mimicking the 'adjuvant' properties of LDV through type I IFN-inducing agents may augment antiretroviral NAb responses by modulating APOBEC3 activity.

\section{Methods}

\section{Mice}

C57BL/6 (B6) and IFNAR KO mice [41], backcrossed for over 15 generations in the B6 background, were purchased from the Jackson Laboratory. mA3 KO mice, generously provided by Dr. Warner Greene, were initially derived from a 129P2 embryonic stem cell gene trap library [10] and backcrossed for 10 generations into B6. The IFNAR and $\mathrm{mA} 3$ genes are located on chromosomes 16 and 15, respectively. To generate IFNAR/mA3 dKO mice, IFNAR $\mathrm{KO}$ and $\mathrm{mA} 3 \mathrm{KO}$ mice were crossed and the progeny genotyped to generate $\mathrm{IFNAR}^{+/-} \mathrm{mA}^{+/-}$ mice. These heterozygous mice were further crossed to obtain IFNAR $^{-1-} \mathrm{mA3}^{-/-}$mice $(\sim 6.25 \%$ by Mendelian genetics). The cohorts described in this study specifically compared IFNAR KO versus IFNAR/mA3 dKO mice less than 6 months of age.

\section{FV infections}

Two FV stocks were used in this study. FV/LDV was in vivo passaged from the FV stock that was used to initially describe Rfv3 [21]. LDV-free FV (or simply FV) was in vitro passaged, and confirmed to have no contaminating LDV [30]. Both stocks were prepared in BALB/c mice and titered as previously described. FV (2000 to 10,000 SFFU) was inoculated intravenously through 
the retroorbital sinus in $300 \mu \mathrm{l}$ RPMI and bleeds or terminal harvests were obtained at either 7 or 28 days post-infection.

\section{Plasma virus infectious titers}

As previously described [17, 18], $5 \mu \mathrm{l}$ of plasma were incubated with Mus dunni cells in a 48-well plate, and after 2 days, F-MuLV DNA copies were measured by quantitative PCR normalized to 100 ng total DNA input.

\section{Plasma viral load quantification}

Viral RNA was extracted from $50 \mu \mathrm{l}$ of plasma using the RNAEasy kit (Qiagen). The isolated RNA was then subjected to a one-step TaqMan reverse transcriptase PCR reaction using FV-specific primers as previously described $[17,18]$. FV copy numbers were determined against a standard curve using T7-transcribed RNA standards.

\section{Flow cytometry}

Splenocytes were stained for the F-MuLV glyco-gag protein using the mAb 34 antibody for $1 \mathrm{~h}$, then stained with anti-mouse IgG1-APC (Columbia Biosciences) for 30 min. An LSR-II flow cytometer (BD Biosciences) was used to capture up to 250,000 events per sample, and Flowjo software (Treestar) was used to analyze the data. Glyco-gag+ cells were gated based on biological controls using uninfected splenocytes (Fig. 2a).

\section{Neutralizing antibody assay}

Serial dilutions of heat-inactivated plasma were combined with a standard amount (50-100 infectious units) of F-MuLV for $1 \mathrm{~h}$ at $37^{\circ} \mathrm{C}$, then the mixture was added onto Mus dunni cells in 48-well plates. After 2 days, F-MuLV titers were detected using an F-MuLV Env-specific monoclonal antibody, mAb 720 [10, 53]. Inhibition curves were constructed by nonlinear regression using the one-site total equation in GraphPad Prism 5.0 [16]. $\mathrm{NAb}$ titers corresponded to the concentration of plasma that resulted in 50\% neutralization compared to the control samples with F-MuLV alone.

\section{FV-specific lgG2b/c titers}

Endpoint titration ELISAs were performed as previously described $[16,25]$. Briefly, serial twofold dilutions of plasma were incubated for $1 \mathrm{~h}$ on 96-well Immulon-4 HBX plates pre-coated with 200 ng native FV virions. After 6 washes with PBS-Tween 10, an equimolar combination of anti-IgG2b and anti-IgG2c antibodies (1:4000; Southern Biotechnology) conjugated to HRP were added, washed, then incubated with TMB substrate. Endpoint titers were calculated as the plasma dilution that corresponded to twice the mean background of wells without plasma added.

\section{Statistical analyses}

Infection and antibody values were log-transformed to normalize the data for analysis using a 2-tailed unpaired Student's $t$ test (GraphPad Prism 5.0).

\section{Additional file}

Additional file 1: Fig. S1. Four-way comparison of NAb responses. $\mathrm{NAb}$ data from WT versus $\mathrm{mA} 3 \mathrm{KO}$ mice from Fig. 3 and IFNAR KO versus IFNAR/mA3 dKO mice from Fig. 4c were analyzed. Inoculum doses were lower in the IFNAR KO background to account for the higher susceptibility of these mouse strains to FV infection. Pairwise analyses were performed using a 2-tailed Student's $t$ test. ${ }^{*} p<0.05 ;{ }^{* *} p<0.01$; NS not significant.

\section{Authors' contributions}

MLS, BSB, MSH and KJHasenkrug designed the research; BSB, STJ, MSH, KG AND KJHeilman performed the experiments; RMK contributed reagents and expertise; MLS, BSB, STJ, MSH, KG and KJHeilman analyzed data; MLS, KJHasenkrug, BSB and MSH wrote the paper; MLS supervised the work. All authors read and approved the final manuscript.

\section{Author details \\ ${ }^{1}$ Department of Medicine, University of Colorado Denver, Aurora, CO, USA. 2 Department of Immunology and Microbiology, University of Colorado Den- ver, Aurora, CO, USA. ${ }^{3}$ Rocky Mountain Laboratories, NIAID, NIH, Hamilton, MT, USA. ${ }^{4}$ Division of Infectious Diseases, University of Colorado Denver, Mail Stop B-168, 12700 E 19th Avenue, Aurora, CO 80045, USA.}

\section{Competing interests}

The authors declare that they have no competing interests.

\section{Ethics approval and consent to participate}

Mice were handled in accordance with the recommendations of the $\mathrm{NIH}$ Guide for the Care and Use of Laboratory Animals and approved by the UCD IACUC committee [permit number B-89715(07)1E].

\section{Funding}

This work was supported by National Institutes of Health (NIH) Grant R01 Al1 16603 (MLS) the University of Colorado Department of Medicine Early Career Scholar Program (MLS), The RNA Bioscience Initiative (MLS) and the Intramural Research Program at the National Institutes of Allergy and Infectious Diseases (KJHasenkrug). MSH was supported in part through the Tim Gill Foundation. STJ is a recipient of the T32 AI007405 University of Colorado Training Program in Immunology Predoctoral Award.

\section{Publisher's Note}

Springer Nature remains neutral with regard to jurisdictional claims in published maps and institutional affiliations.

Received: 26 January 2017 Accepted: 6 April 2017

Published online: 17 April 2017

\section{References}

1. Meager A. The interferons: characterization and application. Germany: Wiley-VCH; 2006.

2. Santiago ML, Greene WC. The role of the Apobec3 family of cytidine deaminases in innate immunity, G-to-A hypermutation and evolution of retroviruses. In: Domingo E, Parrish CR, Holland JJ, editors. Origin and evolution of viruses. London: Academic Press; 2008. p. 183-206.

3. Harris RS, Dudley JP. APOBECs and virus restriction. Virology. 2015;479-480:131-45.

4. Stopak KS, Chiu YL, Kropp J, Grant RM, Greene WC. Distinct patterns of cytokine regulation of APOBEC $3 \mathrm{G}$ expression and activity in 
primary lymphocytes, macrophages, and dendritic cells. J Biol Chem. 2007;282:3539-46.

5. Refsland EW, Stenglein MD, Shindo K, Albin JS, Brown WL, Harris RS. Quantitative profiling of the full APOBEC3 mRNA repertoire in lymphocytes and tissues: implications for HIV-1 restriction. Nucleic Acids Res. 2010;38:4274-84.

6. Goujon C, Malim MH. Characterization of the alpha interferon-induced postentry block to HIV-1 infection in primary human macrophages and T cells. JVirol. 2010;84:9254-66.

7. Sarkis PT, Ying S, Xu R, Yu XF. STAT1-independent cell type-specific regulation of antiviral APOBEC3G by IFN-alpha. J Immunol. 2006;177:4530-40.

8. Malim MH. APOBEC proteins and intrinsic resistance to HIV-1 infection. Philos Trans R Soc Lond B Biol Sci. 2009;364:675-87.

9. Okeoma CM, Lovsin N, Peterlin BM, Ross SR. APOBEC3 inhibits mouse mammary tumour virus replication in vivo. Nature. 2007:445:927-30.

10. Santiago ML, Montano M, Benitez R, Messer RJ, Yonemoto W, Chesebro B, Hasenkrug KJ, Greene WC. Apobec3 encodes Rfv3, a gene influencing neutralizing antibody control of retrovirus infection. Science. 2008;321:1343-6.

11. Takeda E, Tsuji-Kawahara S, Sakamoto M, Langlois MA, Neuberger MS, Rada C, Miyazawa M. Mouse APOBEC3 restricts friend leukemia virus infection and pathogenesis in vivo. J Virol. 2008;82:10998-1008.

12. Low A, Okeoma CM, Lovsin N, de las Heras M, Taylor TH, Peterlin BM, Ross $\mathrm{SR}$, Fan $\mathrm{H}$. Enhanced replication and pathogenesis of Moloney murine leukemia virus in mice defective in the murine APOBEC3 gene. Virology. 2009;385:455-63.

13. Hasenkrug KJ, Dittmer U. Immune control and prevention of chronic Friend retrovirus infection. Front Biosci. 2007:12:1544-51.

14. Best S, Le Tissier P, Towers G, Stoye JP. Positional cloning of the mouse retrovirus restriction gene Fv1. Nature. 1996;382:826-9.

15. Halemano K, Harper MS, Guo K, Li SX, Heilman KJ, Barrett BS, Santiago ML. Humoral immunity in the Friend retrovirus infection model. Immunol Res. 2013;55:249-60

16. Smith DS, Guo K, Barrett BS, Heilman KJ, Evans LH, Hasenkrug KJ, Greene WC, Santiago ML. Noninfectious retrovirus particles drive the APOBEC3/Rfv3 dependent neutralizing antibody response. PLoS Pathog. 2011;7:e1002284

17. Harper MS, Barrett BS, Smith DS, Li SX, Gibbert K, Dittmer U, Hasenkrug KJ, Santiago ML. IFN-alpha treatment inhibits acute Friend retrovirus replication primarily through the antiviral effector molecule Apobec3. J Immunol. 2013;190:1583-90.

18. Li SX, Barrett BS, Harper MS, Heilman KJ, Halemano K, Steele AK, Guo K, Silverman RH, Santiago ML. Ribonuclease $L$ is not critical for innate restriction and adaptive immunity against Friend retrovirus infection. Virology. 2013:443:134-42.

19. Doig D, Chesebro B. Anti-Friend virus antibody is associated with recovery from viremia and loss of viral leukemia cell-surface antigens in leukemic mice. Identification of Rfv-3 as a gene locus influencing antibody production. J Exp Med. 1979;150:10-9.

20. Santiago ML, Smith DS, Barrett BS, Montano M, Benitez RL, Pelanda R, Hasenkrug KJ, Greene WC. Persistent Friend virus replication and disease in Apobec3-deficient mice expressing functional B-cell-activating factor receptor. J Virol. 2011;85:189-99.

21. Chesebro B, Wehrly K. Identification of a non-H-2 gene (Rfv-3) influencing recovery from viremia and leukemia induced by Friend virus complex. Proc Natl Acad Sci USA. 1979:76:425-9.

22. Persons DA, Paulson RF, Loyd MR, Herley MT, Bodner SM, Bernstein A, Correll PH, Ney PA. Fv2 encodes a truncated form of the Stk receptor tyrosine kinase. Nat Genet. 1999;23:159-65.

23. Jelacic TM, Thompson D, Hanson C, Cmarik JL, Nishigaki K, Ruscetti S. The tyrosine kinase sf-Stk and its downstream signals are required for maintenance of friend spleen focus-forming virus-induced fibroblast transformation. J Virol. 2008;82:419-27.

24. Beck-Engeser GB, Winkelmann R, Wheeler ML, Shansab M, Yu P, Wunsche S, Walchhutter A, Metzner M, Vettermann C, Eilat D, et al. APOBEC3 enzymes restrict marginal zone B cells. Eur J Immunol. 2015;45:695-704.

25. Halemano K, Barrett BS, Heilman KJ, Morrison TE, Santiago ML. Requirement for Fc effector mechanisms in the APOBEC 3/Rfv3-dependent neutralizing antibody response. JVirol. 2015;89:4011-4.

26. Halemano K, Guo K, Heilman KJ, Barrett BS, Smith DS, Hasenkrug KJ, Santiago ML. Immunoglobulin somatic hypermutation by APOBEC3/Rfv3 during retroviral infection. Proc Natl Acad Sci USA. 2014;111:7759-64.
27. Li K, Schuler T, Chen Z, Glass GE, Childs JE, Plagemann PG. Isolation of lactate dehydrogenase-elevating viruses from wild house mice and their biological and molecular characterization. Virus Res. 2000;67:153-62.

28. Virgin HW. The virome in mammalian physiology and disease. Cell. 2014;157:142-50

29. Duley AK, Ploquin MJ, Eksmond U, Ammann CG, Messer RJ, Myers L, Hasenkrug KJ, Kassiotis G. Negative impact of IFN-gamma on early host immune responses to retroviral infection. J Immunol. 2012;189:2521-9.

30. Robertson SJ, Ammann CG, Messer RJ, Carmody AB, Myers L, Dittmer U, Nair S, Gerlach N, Evans LH, Cafruny WA, Hasenkrug KJ. Suppression of acute anti-friend virus CD8+ T-cell responses by coinfection with lactate dehydrogenase-elevating virus. J Virol. 2008;82:408-18.

31. Marques R, Antunes I, Eksmond U, Stoye J, Hasenkrug K, Kassiotis G. B lymphocyte activation by coinfection prevents immune control of friend virus infection. J Immunol. 2008;181:3432-40.

32. Ammann CG, Messer RJ, Peterson KE, Hasenkrug KJ. Lactate dehydrogenase-elevating virus induces systemic lymphocyte activation via TLR7dependent IFNalpha responses by plasmacytoid dendritic cells. PLoS One. 2009;: :e6105.

33. Gerlach N, Schimmer S, Weiss S, Kalinke U, Dittmer U. Effects of Type I Interferons on Friend retrovirus infection [Author's correction]. J Virol. 2007:81:6160.

34. Fink K, Lang KS, Manjarrez-Orduno N, Junt T, Senn BM, Holdener M, Akira $\mathrm{S}$, Zinkernagel RM, Hengartner H. Early type I interferon-mediated signals on B cells specifically enhance antiviral humoral responses. Eur J Immunol. 2006;36:2094-105.

35. Finkelman FD, Svetic A, Gresser I, Snapper C, Holmes J, Trotta PP, Katona IM, Gause WC. Regulation by interferon alpha of immunoglobulin isotype selection and lymphokine production in mice. J Exp Med. 1991;174:1179-88.

36. Bayer W, Lietz R, Ontikatze T, Johrden L, Tenbusch M, Nabi G, Schimmer $\mathrm{S}$, Groitl P, Wolf H, Berry CM, et al. Improved vaccine protection against retrovirus infection after co-administration of adenoviral vectors encoding viral antigens and type I interferon subtypes. Retrovirology. 2011;8:75.

37. Le Bon A, Schiavoni G, D'Agostino G, Gresser I, Belardelli F, Tough DF. Type i interferons potently enhance humoral immunity and can promote isotype switching by stimulating dendritic cells in vivo. Immunity. 2001;14:461-70.

38. Michaelides MC, Simms ES. Immune responses in mice infected with lactic dehydrogenase virus. I. Antibody response to DNP-BGG and hyperglobulinaemia in BALB/c mice. Immunology. 1977;32:981-8.

39. Isakov N, Feldman M, Segal S. The mechanism of modulation of humoral immune responses after infection of mice with lactic dehydrogenase virus. J Immunol. 1982:128:969-75.

40. Lavoie TB, Kalie E, Crisafulli-Cabatu S, Abramovich R, DiGioia G, Moolchan K, Pestka S, Schreiber G. Binding and activity of all human alpha interferon subtypes. Cytokine. 2011;56:282-9.

41. Muller U, Steinhoff U, Reis LF, Hemmi S, Pavlovic J, Zinkernagel RM, Aguet M. Functional role of type I and type II interferons in antiviral defense. Science. 1994;264:1918-21.

42. Gerlach N, Schimmer S, Weiss S, Kalinke U, Dittmer U. Effects of type I interferons on Friend retrovirus infection. J Virol. 2006;80:3438-44.

43. Li SX, Barrett BS, Guo K, Kassiotis G, Hasenkrug KJ, Dittmer U, Gibbert K, Santiago ML. Tetherin/BST-2 promotes dendritic cell activation and function during acute retrovirus infection. Sci Rep. 2016;6:20425.

44. Dittmer U, Race B, Peterson KE, Stromnes IM, Messer RJ, Hasenkrug $\mathrm{KJ}$. Essential roles for $\mathrm{CD} 8+\mathrm{T}$ cells and gamma interferon in protection of mice against retrovirus-induced immunosuppression. J Virol. 2002;76:450-4

45. Swanson CL, Wilson TJ, Strauch P, Colonna M, Pelanda R, Torres RM. Type I IFN enhances follicular B cell contribution to the T cell-independent antibody response. J Exp Med. 2010;207:1485-500.

46. Nimmerjahn F, Lux A, Albert H, Woigk M, Lehmann C, Dudziak D, Smith P, Ravetch JV. FcgammaRIV deletion reveals its central role for IgG2a and IgG2b activity in vivo. Proc Natl Acad Sci USA. 2010;107:19396-401.

47. Santiago ML, Benitez RL, Montano M, Hasenkrug KJ, Greene WC. Innate retroviral restriction by Apobec 3 promotes antibody affinity maturation in vivo. J Immunol. 2010;185:1114-23.

48. Okeoma CM, Petersen J, Ross SR. Expression of murine APOBEC3 alleles in different mouse strains and their effect on mouse mammary tumor virus infection. J Virol. 2009:83:3029-38. 
49. Sanville B, Dolan MA, Wollenberg K, Yan Y, Martin C, Yeung ML, Strebel K, Buckler-White A, Kozak CA. Adaptive evolution of Mus Apobec3 includes retroviral insertion and positive selection at two clusters of residues flanking the substrate groove. PLoS Pathog. 2010;6:e1000974.

50. Gibbert K, Schlaak JF, Yang D, Dittmer U. IFN-alpha subtypes: distinct biological activities in anti-viral therapy. Br J Pharmacol. 2013;168:1048-58.

51. Gerlach N, Gibbert K, Alter C, Nair S, Zelinskyy G, James CM, Dittmer U. Anti-retroviral effects of type I IFN subtypes in vivo. Eur J Immunol. 2009;39:136-46.
52. Getahun A, Wemlinger SM, Pratyaydipta R, Santiago ML, van Dyk LF, Cambier JC. Impaired B cell function during viral infections due to PTENmediated inhibition of the PI3K pathway. J Exp Med. 2017;214:931-41.

53. Robertson MN, Miyazawa M, Mori S, Caughey B, Evans LH, Hayes SF, Chesebro B. Production of monoclonal antibodies reactive with a denatured form of the Friend murine leukemia virus gp70 envelope protein: use in a focal infectivity assay, immunohistochemical studies, electron microscopy and western blotting. J Virol Methods. 1991;34:255-71.

\section{Submit your next manuscript to BioMed Central and we will help you at every step:}

- We accept pre-submission inquiries

- Our selector tool helps you to find the most relevant journal

- We provide round the clock customer support

- Convenient online submission

- Thorough peer review

- Inclusion in PubMed and all major indexing services

- Maximum visibility for your research

Submit your manuscript at

www.biomedcentral com/submit 